\title{
Advantages of lung ultrasound in triage, diagnosis and monitoring COVID-19 patients: review
}

\author{
Bianca Emilia Ciurba1, Hédi Katalin Sárközi ${ }^{1,2}$, István Adorján Szabó1,2, Nimród László1, Edith Simona \\ Ianosi ${ }^{1,2}$, Maria lanosi $2^{\star}$, Gabriela Jimborean ${ }^{1,2}$ \\ 1. Mures County Clinical Hospital, Romania \\ 2. George Emil Palade University of Medicine, Pharmacy, Science, and Technology of Targu Mures, Romania
}

Over the last decades, especially during the COVID-19 pandemic period, lung ultrasound (LUS) gained interest due to multiple advantages: radiation-free, repeatable, cost-effective, portable devices with a bedside approach. These advantages can help clinicians in triage, in positive diagnostic, stratification of disease forms according to severity and prognosis, evaluation of mechanically ventilated patients from Intensive Care Units, as well as monitoring the progress of COVID-19 lesions, thus reducing the health care contamination. LUS should be performed by standard protocol examination. The characteristic lesions from COVID-19 pneumonia are the abolished lung sliding, presence of multiple and coalescent B-lines, disruption and thickening of pleural line with subpleural consolidations. LUS is a useful method for post-COVID-19 lesions evaluation, highlight the remaining fibrotic lesions in some patients with moderate or severe forms of pneumonia.

Keywords: lung ultrasound, COVID-19, pleura, diagnosis

Received 28 April 2021 / Accepted 2 June 2021

\section{Introduction}

Over the last decades, lung ultrasound (LUS) successfully entered into the international protocols for diagnosis of respiratory diseases (especially in emergency and for pleural damage evaluation). The first protocol was described by Daniel Liechtenstein in 2008, named the BLUE protocol, which identifies the cause of dyspnea and respiratory failure, with a bedside approach [1]. Since then, LUS has gained more and more interest even during the COVID-19 pandemic, because of its several advantages. Nowadays we have access to portable US devices (convenient for emergency medicine or ICU). LUS technique is repeatable and more affordable than computerized tomography (CT). In this work we aimed to review the utility and advantages of LUS in triage, suspicion raises diagnosis and monitoring the COVID-19 patients and highlighted the suggestive LUS findings associated with SARS-COV2 pneumonia.

What are the advantages of LUS in the COVID-19 pandemic period?

First of all, and the most important advantage in our opinion is that LUS is a non-irradiant, noninvasive, repeatable method, thus it can be suitable for the evaluation of severely ill patients, in the emergency room or in ICU and even it can be used in pregnant women. LUS can be performed at the patient's bedside, integrates real-time imaging into the clinical decision, and does not claim special patient preparation which is important in the rapid triage of the patients with clinical suspicion of COVID-19.

In the last two years, it has been developed portable devices, even with wireless US probes that can be covered

* Correspondence to: Maria lanosi

E-mail: maria99ianosi@yahoo.com with sterile sheath and easily decontaminated before and after the procedure. All this contributes to reducing the crossover contamination of the healthcare providers and decreases the time of the investigation. Another advantage was described in 2009 during the influenza epidemic (H1N1) when it has been demonstrated that LUS could differentiate viral pneumonia from bacterial pneumonia [2].

LUS can identify pulmonary lesions in the early phase of the disease and evaluate the progression of COVID-19 lesions during the hospitalization along with patients' stratification by severity and prognosis. LUS may also highlight the remaining fibrotic lesions in some patients with moderate or severe forms of pneumonia and permits long-term follow-up.

Even though thoracic CT is considered the "gold standard" imaging method in diagnosing COVID-19 pneumonia, CT scans could, in several moments, be replaced with LUS examination, because CT is still irradiant and access to the CT of the high infectious patients is difficult. Thus, CT has a lack of repeatability in a short interval of time. More than that, many studies reported that LUS has comparable diagnostic accuracy with CT findings and superior to chest X-ray [3,29].

Other advantages of LUS and potential users are in triage for patients with acute respiratory failure (a prompt LUS examination can be done using BLUE protocol in less than 3 minutes) [4-6]. LUS is a complement diagnostic method in patients with high clinical suspicion of COVID-19 with inconclusive changes on chest $\mathrm{x}$-ray and a negative result on RT-PCR nasopharyngeal swab test. Because patients' transportation to radiology departments for CT evaluation is difficult or not possible (like in ICU), LUS associated with echocardiography is more effective in evalu- 
ation and optimization of the treatment $[7,8]$. For critically ill patients (with bilateral and extensive B-lines and pleural irregularities) LUS is able to evaluate the need for mechanical ventilation and post procedures and detect complications like pneumothorax or pleural effusion [9-15].

\section{LUS technique}

LUS should be performed by a standard protocol of examination and two types of probes are suitable for examination $[16,17]$. The linear probe with high frequencies [9-12 $\mathrm{MHz}$ ), allows evaluation of superficial structure focusing on pleural line and superficial parenchyma. The convex probe with lower frequencies $(3-5 \mathrm{MHz})$ but higher capacity of penetration allows better visualization of deep parenchymal structures and the diaphragm and it is suitable for obese patients [33].

In the last years, US machines have incorporated the lung preset (special application) with a depth setting of 6-10 cm for full appreciation of the extension of B-lines. There are two possible approaches, scanning longitudinally when the probe is placed perpendicular to the ribs, and transverse/oblique approach when the probe is placed horizontally along with the intercostal space. The oblique approach is better because allows visualizing a larger part of the pleural line, which is not interrupted by the rib shadows. LUS should be performed in sitting position [33]. If the patient can't sit up, LUS must be performed in a supine position with the head of the bed elevated. Critically ill patients with mechanical ventilation can be evaluated by tilting the patient on the side. It has been reported in many studies that a more comprehensive examination in the 16 thoracic zones has better accuracy in diagnosing COVID-19 lesions. For example, dividing the chest wall into 4 regions on both sides by 5 anatomical lines (parasternal, anterior and posterior axillary, scapular and paravertebral), every region with further division into an upper and lower area [18].

Which are the most frequent and characteristic LUS patterns found in COVID-19?

The normal lung with well aeration is characterized by A-lines, horizontal equidistant and repetitive reverberation artifacts, parallel to the pleural line. As the air decrease and the fluid increase in the subpleural interlobular septa, Bline artifacts start to appear.

B-lines are vertical hyperechoic laser-like rays arising from the pleural line and extended to the edge of the screen, which move with respiration (a sign called lungsliding). The B-line pattern is the most characteristic sign in COVID-19 with different varieties (separated B-lines, focal, multifocal, coalescent, "rocket sign", "light beam"), reported by most of the studies, with a predominantly posterior and inferior distribution [19-24]. The B-lines can coexist with A-lines, in the context of good aeration, called spared areas. When the aeration of the lung progressively decreases, the B-lines increase and become coalescent. When the extension of B-lines covered the entire rib space and persists during the entire respiratory cycle the "white lung" sign is described (suggesting very severe lesions and lack of ventilation) [24]. Some of these featured are presented in figure 1 (images taken from our patients from Pulmonology Clinic Târgu Mureș during COVID-19 pneumonia).

Another specific pattern found in COVID-19 patients is pleural thickening with disruption and subpleural consolidation, named "shred sign" [25]. Because the increase of thromboembolic events in COVID-19 was reported, small subpleural consolidation with disruption of the pleura may indicate subpleural microinfarctions and Doppler function of LUS or "contrast-enhanced US technique" should help us for a clear diagnostic [26]. Subpleural small consolida-
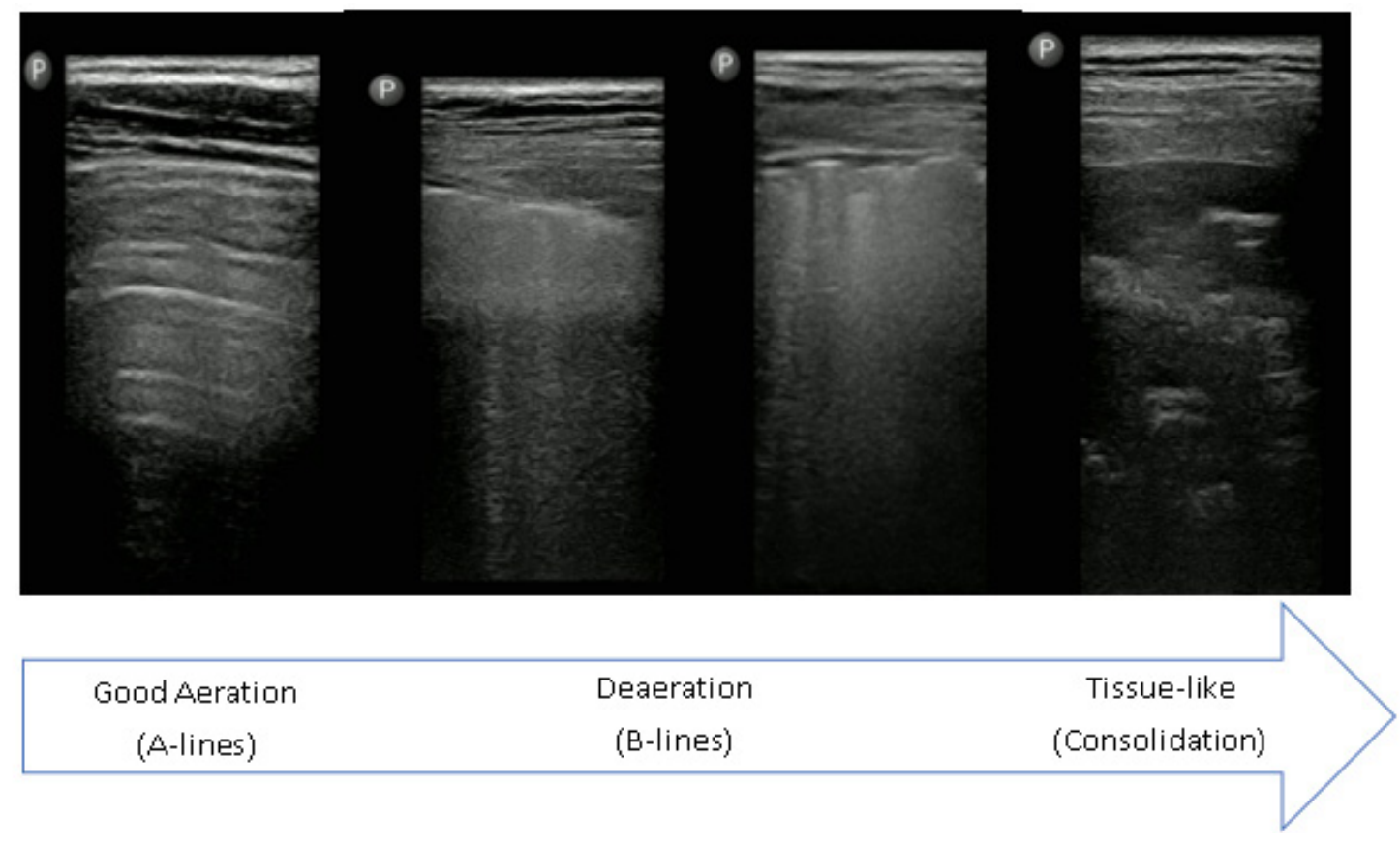

Fig. 1. Our experience regarding the LUS characteristic lesions found in COVID-19 patients (we used a linear probe with lung preset). 
tions can progress into large poorly vascularized or avascular consolidation with dynamic or static air bronchogram, which can indicate a bacterial superinfection or a severe evolution (total absence of ventilation) [27]. Pleural effusion was an extremely rare sign associated with COVID-19 lesions, reported by lots of studies $[19,27,18]$.

Findings of these characteristic LUS patterns, even in the absence of RT PCR results, must help clinicians to take the decision of prompt isolation and treatment of the pulmonary lesions.

A recent Spanish study conducted in the Internal Medicine department by specialists (with long experience in LUS), examined 130 patients with COVID-19 using LUS in the first 24 hours after admission, during the hospitalization and at discharge. They examined 12 areas (2 anterior, 2 lateral and 2 posterior for each lung) and quantified the lung lesions by a score between 0 and 4, called „Lung Ultrasound Zaragoza Score” (LUZ). This score corresponds to the characteristic of LUS findings described anteriorly and assigns the following score: a). 0 point for the presence of A-lines with a normal pleural line; $b$ ). 1 point for A-lines coexisting with isolated and small B-lines; c). 2 points for disappearing of A-lines, multiple B-lines alternating with normal parenchymal areas and pleural line thickening with small „bites”; d). 3 points for coalescent B-lines that fill the entire intercostal space (waterfall pattern) and e). 4 points for broken pleural line with subpleural consolidations (1 to $1.5 \mathrm{~cm}$ deep). The study reported that a LUZ-score of more than 22 predicted the severity of lung involvements with the necessity to patient's transfer in the ICU and the need for invasive mechanical ventilation [29].

\section{"Long COVID-19" and LUS follow up}

The role of LUS in the evaluation of residual lung injury after acute COVID-19 is a subject in the progress of research. Clinicians noticed persisting symptoms and organ dysfunction in numerous patients who have recovered after COVID-19, especially in those who had severe and extensive pulmonary involvements. At 6 months after the SARS COV2 infection onset, a significant number of patients remain with persistent fatigue, dyspnea, muscle weakness, sleep difficulties, with ground glass opacities at high-resolution CT [34,35]. A lot of patients still required supplemental oxygen. A COVID-19 follow-up study described that after one month from the first LUS evaluation, subpleural consolidations on LUS predict the lasting symptoms and a more severe course of the residual pulmonary lesions [35]. Another study which evaluated the lung lesions after four months post-hospital discharge on a cohort of 127 COVID-19 patients, found that 15 patients developed interstitial lung disease (fibrosis), findings that are compatible with non-specific interstitial pneumonia (NSIP) on high-resolution CT and lung biopsy [36]. In a previous study, has been highlighted that 21 out of 49 COVID-19 patients developed lung fibrosis after 30 days from hospital admission [38]. This subject of long postCOVID evolution and complications is debatable and needs long-term follow-up to determine if those lesions could be influenced or they are irreversible. As a sign of recovery (with increasing aeration in the lung) described the reappearance of A-lines [37].

\section{Differential diagnosis of COVID-19 ultrasound patterns}

These patterns described anterior are not specific only for COVID-19 lesions and could be found in several pathologies. The ultrasound differential diagnosis of lesions and distribution can be seen in Table 1 .

\section{Conclusions}

LUS plays a key role during the COVID-19 pandemic, as it is an affordable, non-irradiant, portable method and it has the potential to improve the management of COVID-19 patients. The use of portable LUS has to become a common practice in emergency departments for triage and patients' stratification and in pulmonology and ICU departments for diagnosing the characteristic COVID-19 abnormalities and monitoring the disease evolution. LUS could be in the future an accessible tool for monitoring the post COVID-19 status.

Table 1. Differential diagnosis of COVID-19 ultrasound patterns

\begin{tabular}{|c|c|c|c|c|}
\hline & COVID-19 & Cardiogenic pulmonary oedema [31] & Bacterial Pneumonia [32] & Interstitial Lung disease $[33,34]$ \\
\hline Distribution & Patchy lesions with spared areas & $\begin{array}{l}\text { Diffuse and bilateral lesions, without } \\
\text { spared areas }\end{array}$ & Focal & $\begin{array}{l}\text { Predominant Inferior, at lung } \\
\text { bases }\end{array}$ \\
\hline Lung Sliding & Abolished & Present & Abolished & Abolished \\
\hline B-lines & $\begin{array}{l}\text { Non-gravity related. } \\
\text { Focal, separated and more often } \\
\text { coalescent (white lung) }\end{array}$ & $\begin{array}{l}\text { Homogenous, } \\
\text { gravity-related. } \\
\text { Usually separated. } \\
\text { Coalescent in severe cases }\end{array}$ & $\begin{array}{l}\text { Not frequent } \\
\text { Only in the case of focal } \\
\text { interstitial syndrome }\end{array}$ & $\begin{array}{l}\text { Usually separated, but also } \\
\text { coalescent in severe cases }\end{array}$ \\
\hline Pleural line & Irregular, thickened, discontinued & Regular and thin, no interruption seen & $\begin{array}{l}\text { Not visible because of the } \\
\text { consolidation }\end{array}$ & Always irregular \\
\hline Consolidation & $\begin{array}{l}\text { Subpleural consolidation(small and } \\
\text { peripheral) } \\
\text { Larger and deeper consolidation } \\
\text { less frequent (seen in critically ill } \\
\text { patients with bacterial suprainfec- } \\
\text { tion) }\end{array}$ & $\begin{array}{l}\text { Not present, only when a large } \\
\text { pleural effusion causes compressive } \\
\text { atelectasis }\end{array}$ & $\begin{array}{l}\text { Large hyperechoic, "tissue- } \\
\text { like" image with dynamic or } \\
\text { static bronchogram }\end{array}$ & Rarely/not present \\
\hline Pleural effusion & Rare & $\begin{array}{l}\text { Frequent usually bilateral (transudate- } \\
\text { anechoic) }\end{array}$ & $\begin{array}{l}\text { Usually small, with plankton } \\
\text { sign (exudate-hyperechoic ) }\end{array}$ & Usually small \\
\hline
\end{tabular}




\section{Acknowledgements and funding}

The research received no external funding. The authors received no financial support for the research, authorship and/or publication of this article.

\section{Conflict of Interest}

The authors declared that is no potential conflicts of interest with respect to the research, authorship and/or publication of this article.

\section{Authors' contribution}

C.B., J.G. (Conception of the work; Data interpretation; Formal Analysis; Methodology; Project administration; Drafting and critical revise; Resources; Writing - review \& editing; Agreement to be accountable for all aspects of the work).

S.H., S.I., L.N., I.M., I.E. (Conception of the work; Data interpretation; Formal Analysis; Methodology; Drafting and critical revise; Resources; Agreement to be accountable for all aspects of the work).

\section{References}

1. Liechtenstein DA, Meziere GA. Relevance of Lung Ultrasound in the Diagnosis of Acute Respiratory Failure, The BLUE Protocol. Chest. 2008;134:117-125.

2. Tsung JW, Kessler DO, Shah VP. Prospective application of clinicianperformed lung ultrasonography during the 2009 H1N1 influenza A pandemic: distinguishing viral from bacterial pneumonia. Crit Ultrasound J. 2012;4-16.

3. Lieveld A W E, Kok B, Schuit F H, et al. Diagnosing COVID-19 pneumonia in a pandemic setting: Lung Ultrasound versus CT (LUVCT) - a multicenter, prospective, observational study. ERJ Open Res. 2020; 6(4):00539-2020.

4. Volpicelli G, Gargani L. Sonographic signs and patterns of COVID-19 pneumonia. Ultrasound J. 2020;12(1):22

5. Piliego C, Strumia A, Stone MB, Pascarella G. The UltrasoundGuided Triage: A New Tool for Prehospital Management of COVID-19 Pandemic. Anesth Analg. 2020;131(2):e93-e94.

6. Lichtenstein DA. Lung ultrasound in the critically ill. Ann Intensive Care. 2014:4(1):1.

7. Jg C, Butcher C, Sola A. British Heart Diseases Natural history and prognostic value of ultrasound lung artefacts ("comet tail" sign) in patients admitted to hospital for worsening heart failure. $\mathrm{Br} \mathrm{J}$ Heart Dis. 2018;1:112-118.

8. Volpicelli G, Elbarbary M, Blaivas M, et al. International evidence-based recommendations for point-of-care lung ultrasound. Intensive Care Med. 2012;38:577-591.

9. Volpicelli $G$, Elbarbary $M$, Blaivas $M$, Lichtenstein DA, et al. International evidence-based recommendations for point-of-care lung ultrasound. Intensive Care Med. 2012;38(4):577-591.

10. Lichtenstein DA, Mezière $\mathrm{G}$, Lascols $\mathrm{N}$, et al. Ultrasound diagnosis of occult pneumothorax. Crit Care Med. 2005;33(6):1231-1238.

11. Lichtenstein D. Whole body ultrasonography in the critically ill. Springer 2010, eBook.

12. Tusman G, Acosta CM, Costantini M. Ultrasonography for the assessment of lung recruitment maneuvers. Crit Ultrasound J. 2016;8(1):8

13. Bouhemad $B$, Brisson $H$, Le-Guen $M$, et al. Bedside ultrasound assessment of positive end-expiratory pressure-induced lung recruitment. Am J Respir Crit Care Med. 2011;183:341-7.

14. Bello G, Blanco P. Lung ultrasonography for assessing lung aeration in acute respiratory distress syndrome: a narrative review. J Ultrasound Med. 2019:38:27-37.

15. Raheja R, Brahmavar M, Joshi D, et al. Application of lung ultrasound in critical care setting: a review. Cureus.2019;11:e5233.

16. Soldati $G$, Smargiassi $A$, Inchingolo $R$, et al. Proposal for International Standardization of the Use of Lung Ultrasound for Patients With COVID-19. J Ultrasound Med. 2020;9999:1-7.

17. Gargani L, Soliman-Aboumarie H, Volpicelli G, et al. Why, when and how to use lung ultrasound during the COVID-19 pandemic: enthusiasm and caution. Eur Heart J Cardiovasc Imaging. 2020;1-8.

18. Szabo I A, Agoston G, Varga A, Cotoi O S, Frigy A. Pathophysiological background and clinical practice of lung ultrasound in COVID-19 patients: a short review. Anatol J Cardiol. 2020;24:76-80.

19. Fonsi Battista G, Sapienza P, Brachini G, et al. Is Lung Ultrasound Imaging a Worthwhile Procedure for Severe Acute Respiratory Syndrome Coronavirus 2 Pneumonia Detection. J Ultrasound Med. 2020:9999:1-11.

20. Speidel V, Conen A, Gisler V, et al. Lung assessment with Point-ofCare Ultrasound in Respiratory Coronavirus Disease (COVID-19):a prospective cohort study. Ultrasound Med Biol. 2021;47( 4):896-901.

21. Rizzetto F, Perillo N, Artioli D, Travaglini F, et al. Correlation between lung ultrasound and chest CT patterns with estimation of pulmonary burden in COVID-19 patients. Eur J Radiol. 2021;138:109650.

22. Zanforlin A, Strapazzon G, Falk M, et al. Lung Ultrasound in the Emergency Department for Early Identification of COVID-19 Pneumonia. Respiration. 2021;100(2):145-153.

23. Yasukawa K, Minami T, Boulware D, et al. Point-of-Care Lung Ultrasound for COVID-19: Findings and Prognostic Implications From 105 Consecutive Patient. J Intensive Care Med. 2021;36(3):334-342.

24. Secco G, Delorenzo M,Salinaro F,et.al. Lung ultrasound presentation of COVID-19 patients: phenotypes and correlations. Intern Emerg Med. 2021;1:1-11.

25. Zhao L, Yu K, Zhao Q, Tian R, et al. Lung Ultrasound Score in evaluating the severity of Coronavirus Disease 2019 (COVID-19) Pneumonia. Ultrasound in Med Biol. 2020;46(11):2938-2944.

26. Yusuf TG, Wong A, Rao D, et al. The use of contrast-enhanced ultrasound in COVID-19 lung imaging. J Ultrasound. 2020;1-5.

27. Seiler C, Klingberg C, Hardstedt M. Lung Ultrasound for Identification of Patients Requiring Invasive Mechanical Ventilation in COVID-19. J Ultrasound Med.2021;9999:1-13.

28. Nouvenne A, Zani D M, Milanese G, et al. Lung Ultrasound in COVID-19 Pneumonia: Correlations with Chest CT on Hospital admission. Respiration.2020;99:617-624.

29. Garcia-Rubio J, Lopez-Gimenez I, Horna-Garces V, et al. Point-of-care lung ultrasound assessment for risk stratification and therapy guiding in COVID-19 patients. A prospective non-interventional study. Eur Respir J. 2021;2004283.

30. Gargani L, Frassi F, Soldati G, et al. Ultrasound lung comets for the differential diagnosis of acute cardiogenic dyspnoea: a comparison with natriuretic peptides. Eur J Heart Fail. 2008;10:70-77.

31. Stadler JAM, Andronikou S, Zar HJ. Lung ultrasound for the diagnosis of community-acquired pneumonia in children. Pediatr Radiol. 2017:47(11):1412-1419.

32. Gargani L, Doveri M, D'Errico L, et al. Ultrasound lung comets in systemic sclerosis: a chest sonography hallmark of pulmonary interstitial fibrosis. Rheumatology (Oxford). 2009;48:1382-1387.

33. Jimborean G, lanoși ES, Nemes RM, Toma TP. Basic thoracic ultrasound for the respiratory physician. Pneumologia. 2015;64(3):12-8.

34. Huang $C$, Huang $L$, Wang $Y$, et al. 6-month consequences of COVID-19 in patients discharged from hospital: a cohort study. Lancet. 2021;397:220-3.

35. Mafort TT, Rufino R, Henrique da Costa C, et al. One-month outcomes of patients with SARS-CoV-2 infection and their relationships with lung ultrasound signs. Ultrasound J. 2021:13-19.

36. Alharthy A, Abuhamdah M, Balhamar A, et al. Residual Lung Injury in Patients Recovering From COVID-19 Critical Illness: A Prospective Longitudinal Point-of-Care Lung Ultrasound Study. J Ultrasound Med. 2020:9999:1-16.

37. Sultan LR, Sehgal C M. A Review of early experience in Lung Ultrasound in the diagnosis and management of COVID-19. Ultrasound in Med Biol. 2020;46(9):2530-2545.

38. Lei P, Fan B, Mao J, Wei J, Wang P. The progression of computed tomographic (CT) images in patients with coronavirus disease (COVID-19) pneumonia. J Infect.2020;80:e30-e31. 